\title{
Balanced electronic detection of displacement in nanoelectromechanical systems
}

\author{
K. L. Ekinci, ${ }^{a)}$ Y. T. Yang, X. M. H. Huang, and M. L. Roukes \\ Condensed Matter Physics 114-36, California Institute of Technology, Pasadena, California 91125
}

(Received 8 March 2002; accepted for publication 25 July 2002)

\begin{abstract}
We describe a broadband radio frequency balanced bridge technique for electronic detection of displacement in nanoelectromechanical systems (NEMS). With its two-port actuation-detection configuration, this approach generates a background-nulled electromotive force in a dc magnetic field that is proportional to the displacement of the NEMS resonator. We demonstrate the effectiveness of the technique by detecting small impedance changes originating from NEMS electromechanical resonances that are accompanied by large static background impedances at very high frequencies. This technique allows the study of important experimental systems such as doped semiconductor NEMS and may provide benefits to other high frequency displacement transduction circuits. (C) 2002 American Institute of Physics. [DOI: 10.1063/1.1507833]
\end{abstract}

The recent efforts to scale microelectromechanical systems (MEMS) down to the submicron domain ${ }^{1}$ have opened up an active research field. The resulting nanoelectromechanical systems (NEMS) with fundamental mechanical resonance frequencies reaching into the microwave bands are suitable for a number of important technological applications. Experimentally, they offer potential for accessing interesting phonon mediated processes and the quantum behavior of mesoscopic mechanical systems.

Among the most needed elements for developing NEMS based technologies - as well as for accessing interesting experimental regimes - are broadband, on-chip transduction methods sensitive to subnanometer displacements. While displacement detection at the scale of MEMS has been successfully realized using magnetic, ${ }^{2}$ electrostatic ${ }^{3,4}$ and piezoresistive $^{5}$ transducers through electronic coupling, most of these techniques become insensitive at the submicron scales. Moreover, the attractive electronic two-port actuation-detection configuration of most MEMS devices becomes hard to realize at the scale of NEMS, due to the unavoidable stray couplings encountered with the reduced dimensions of NEMS.

An on-chip displacement transduction scheme that scales well into the NEMS domain and offers direct electronic coupling to the NEMS displacement is magnetomotive detection. 6,7 Magnetomotive reflection measurements as shown schematically ${ }^{8}$ in Fig. 1(a) have been used extensively. ${ }^{6,7,9}$ Here, the NEMS resonator is modeled as a parallel $R L C$ network with a mechanical impedance, $Z_{m}(\omega)$, a two-terminal dc coupling resistance, $R_{e}$, and mechanical resonance frequency, $\omega_{0}$. When driven at $\omega$ by a source with impedance $R_{s}$, the voltage on the load, $R_{L}$, can be approximated as

\footnotetext{
a) Author to whom correspondence should be addressed; present address: Aerospace \& Mechanical Engineering Dept., Boston University, Boston, MA 02215; electronic mail: ekinci@bu.edu
}

$$
\begin{aligned}
V_{0}(\omega) & \simeq V_{\text {in }}(\omega) \frac{R_{e}+Z_{m}(\omega)}{R_{L}+\left[R_{e}+Z_{m}(\omega)\right]} \\
& \simeq V_{\text {in }}(\omega) \frac{R_{e}+Z_{m}(\omega)}{R_{L}+R_{e}} .
\end{aligned}
$$

Here, $R_{L}=R_{S}=50 \Omega$. We have made the approximation that $R_{e} \gg\left|Z_{m}(\omega)\right|$, as is the case in most experimental systems. Apparently, the measured electromotive force (EMF) due to the NEMS displacement proportional to $Z_{m}(\omega)$ is embedded in a background close to the drive voltage amplitude, $\left|V_{o}\right|$ $\sim\left|V_{\text {in }}\right|-20 \log R_{e} /\left(R_{L}+R_{e}\right) \mathrm{dB} .{ }^{10}$ This facilitates the definition of a useful parameter at $\omega=\omega_{0}$, the detection efficiency, $S / B$, as the ratio of the signal voltage to the background. For the reflective, one-port magnetomotive measurement of Fig. $1(\mathrm{a}), S / B=Z_{m}\left(\omega_{0}\right) / R_{e}=R_{m} / R_{e}$, indicating some shortcomings. First, detection of the EMF becomes extremely challenging, when $R_{e} \gg R_{m}$, i.e., in unmetallized NEMS devices or metallized high frequency NEMS (small $R_{m}$ ). Second, the voltage background prohibits the use of the full dynamic range of the detection electronics. As mentioned above, a two-port actuation-detection configuration cannot remedy these problems due to the stray electronic coupling between the ports. ${ }^{11}$

The balanced circuit shown in Fig. 1(b) with a NEMS resonator on one side of the bridge and a matching resistor of resistance, $R=R_{e}+\Delta R$ on the other side, is designed to improve $S / B$. The voltage, $V_{0}(\omega)$ at the readout (RO) port is nulled for $\omega \neq \omega_{0}$, by applying two $180^{\circ}$ out of phase voltages to the Drive 1 (D1) and Drive 2 (D2) ports in the circuit. We have found that the circuit can be balanced with exquisite sensitivity, by fabricating two identical doubly clamped beam resonators on either side of the balance point (RO), instead of a resonator and a matching resistor, as shown in Fig. 1(c). In such devices, we almost always obtained two well-separated mechanical resonances, one from each beam resonator, with $\left|\omega_{2}-\omega_{1}\right| \gg \omega_{i} / Q_{i}$ where $\omega_{i}$ and $Q_{i}$ are the resonance frequency and the quality factor of resonance of the resonators $(i=1,2)$ (see Fig. 3). This indicates that in the vicinity of either mechanical resonance, the system is well 

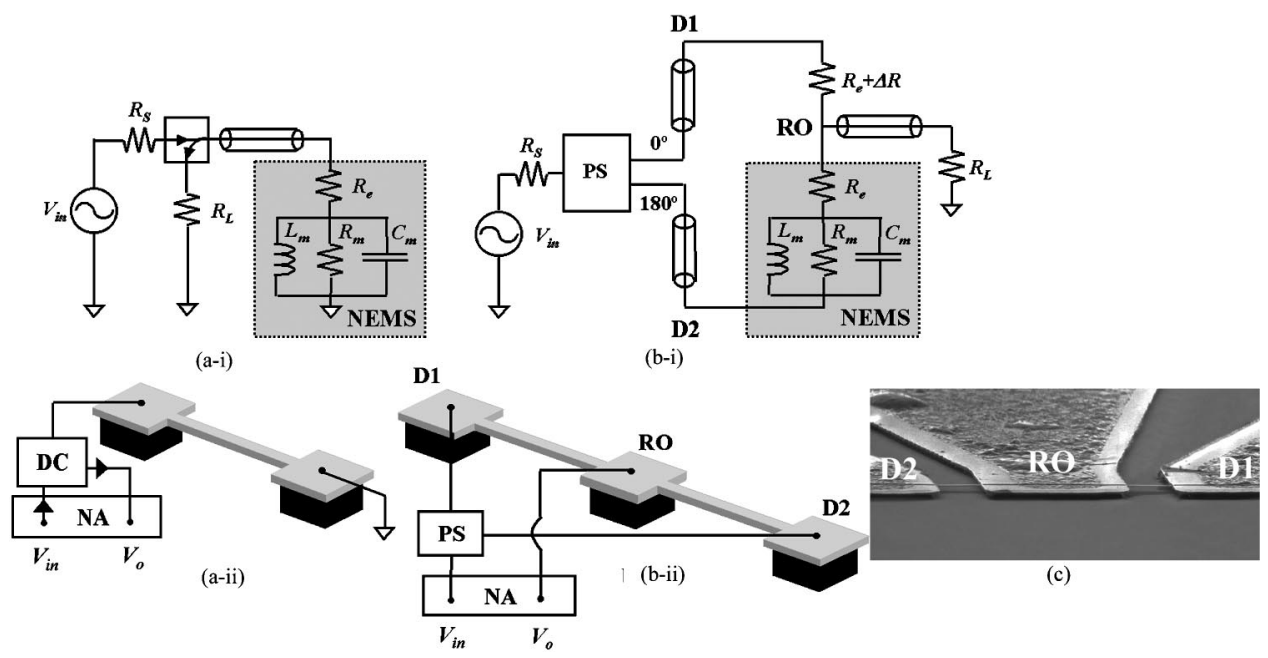

FIG. 1. Schematic diagrams for the magnetomotive reflection (a) and bridge measurements (b). In both measurements, a network analyzer (NA) supplies the drive voltage, $V_{\text {in }}$. In the reflection measurements (a) a directional coupler (DC) is implemented to access the reflected signal from the device. In the bridge measurements (b) $V_{\text {in }}$ is split into two out of phase components by a power splitter (PS) before it is applied to ports D1 and D2. (c) Scanning electron micrograph of a representative bridge device, made out of an epitaxially grown wafer with 50-nm-thick $n+$ GaAs and 100-nm-thick intrinsic GaAs structural layers on top of a 1- $\mu \mathrm{m}$-thick AlGaAs sacrificial layer. The doubly clamped beams with dimensions of $8 \mu \mathrm{m}(L) \times 150 \mathrm{~nm}(w) \times 500 \mathrm{~nm}(t)$ at the two arms of the bridge have in plane fundamental flexural mechanical resonances at $\sim 35 \mathrm{MHz}$. D1, D2, and RO ports on the device are as shown.

described by the mechanical resonator-matching resistor model of Fig. 1(b). We attribute this behavior to the high $Q$ factors $\left(Q \geqslant 10^{3}\right)$ and the extreme sensitivity of the resonance frequencies to local variations of parameters during the fabrication process.

First, to clearly assess the improvements, we compared reflection and balanced bridge measurements of the fundamental flexural resonances of doubly clamped beams patterned from $n^{+}$(B-doped) $\mathrm{Si}$ as well as from $n^{+}$(Si-doped) GaAs. Electronic detection of mechanical resonances of these types of NEMS resonators have proven to be challenging, ${ }^{12}$ since for these systems $R_{e} \geqslant 2 \mathrm{k} \Omega$ and $R_{m}$ $\ll R_{e}$. Nonetheless, with the bridge technique we have detected fundamental flexural resonances in the $10 \mathrm{MHz}<f_{0}$ $<85 \mathrm{MHz}$ range for $n^{+} \mathrm{Si}$ resonators and in the $7 \mathrm{MHz}$ $<f_{0}<35 \mathrm{MHz}$ range for $n^{+}$GaAs beams. In all our measurements, the paradigm that $R_{m} \ll R_{e}$ remained true as $R_{m}$ $\leqslant 10 \Omega$ and $2 \mathrm{k} \Omega<R_{e}<20 \mathrm{k} \Omega$. Here, we focus on our results from $n^{+} \mathrm{Si}$ beams. These were fabricated from a B-doped Si on insulator wafer, with Si layer and buried oxide layer thicknesses of 350 and $400 \mathrm{~nm}$, respectively. The doping was done at $950^{\circ} \mathrm{C}$. The dopant concentration was estimated as $N_{a} \approx 6 \times 10^{19} \mathrm{~cm}^{-3}$ from the sample sheet resistance, $R_{\square} \approx 60 \Omega{ }^{13}$ The fabrication of the actual devices involved optical lithography, electron beam lithography, and lift-off steps followed by anisotropic electron cyclotron resonance plasma and selective HF wet etches. ${ }^{7,9,12}$ The electromechanical response of the bridge was measured in a magnetic field generated by a superconducting solenoid. Figure 2(a) shows the response of a device with dimensions $15 \mu \mathrm{m}(L) \times 500 \mathrm{~nm}(w) \times 350 \mathrm{~nm}(t)$ and with $R_{e} \approx 2.14 \mathrm{k} \Omega$, measured in the reflection (upper curves) and bridge configurations for several magnetic field strengths. The device has an in plane flexural resonance at $25.598 \mathrm{MHz}$ with a $Q \approx 3 \times 10^{4}$ at $T \approx 20 \mathrm{~K}$. With $\Delta R \sim 10 \Omega$ a background reduction of a factor of $\sim 200 \sim R_{e} / \Delta R$ was obtained in the bridge measurements (see analysis below). Figure 2(b) shows a measurement of the broadband transfer functions for both configurations for comparable drives at zero magnetic Downloaded 25 Feb 2006 to 131.215.240.9. Redistribution subject field. Notice the dynamic background reduction in the relevant frequency range.

Bridge measurements also provided benefits in the detection of electromechanical resonances from metallized VHF NEMS. These systems generally possess high $R_{e}$ and $R_{m}$ diminishes quickly as the resonance frequencies increase. Here, we present from our measurements on doubly clamped $\mathrm{SiC}$ beams embedded within the bridge configuration. These beams were fabricated with top metallization layers using a process described in detail. ${ }^{9}$ For such beams with $R_{e}$ $\sim 100 \Omega$ and $R_{m} \leqslant 1 \Omega$, we were able to detect mechanical flexural resonances deep into the VHF band. Figure 3(a) depicts a data trace of the in plane flexural mechanical resonances of two $2 \mu \mathrm{m}(L) \times 150 \mathrm{~nm}(w) \times 80 \mathrm{~nm}(t)$ doubly clamped $\mathrm{SiC}$ beams. Two well-separated resonances are extremely prominent at 198.00 and $199.45 \mathrm{MHz}$, respectively, with $Q \sim 10^{3}$ at $T \sim 4.2 \mathrm{~K}$. The broadband response from the same device is plotted in Fig. 3(b). A reflection measurement in the vicinity of the mechanical resonance frequency of this system would give rise to an estimated background on the order, $\left|V_{o} / V_{\text {in }}\right| \sim-20 \mathrm{~dB}$ (Ref. 10), making the detection of the resonance extremely challenging.

Figure 1(b) depicts our analysis of the bridge circuit. The voltage at point $\mathrm{RO}$ in the circuit can be determined as ${ }^{14}$

$$
\begin{aligned}
V(\omega) & =-\frac{V_{\text {in }}(\omega)\left[\Delta R+Z_{m}(\omega)\right]}{\left(Z_{m}(\omega)+\Delta R\right)\left(1+R_{e} / R_{L}\right)+R_{e}\left(2+R_{e} / R_{L}\right)} \\
& =-\frac{V_{\text {in }}(\omega)}{Z_{\text {eq }}^{\prime}(\omega)}\left[\Delta R+Z_{m}(\omega)\right]
\end{aligned}
$$

by analogy to Eq. (1). At $\omega=\omega_{0}, S / B=R_{m} / \Delta R$. Given that $\Delta R$ is small, the the background is suppressed by a factor of order $R_{e} / \Delta R$, as compared to the one-port case [Fig. 2(a)]. At higher frequencies, however, the circuit model becomes imprecise as is evident from the measurements of the transfer function. Capacitive coupling becomes dominant between D1, D2, and RO ports as displayed in Fig. 2(b), and this acts to reduce the overall effectiveness of the technique. With to AIP license or copyright, see http://apl.aip.org/apl/copyright.jsp 

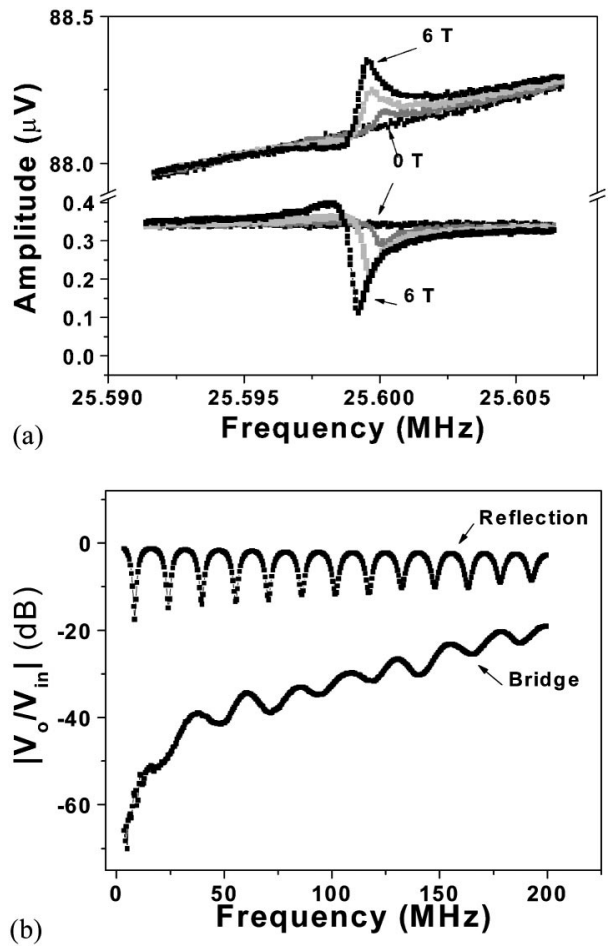

FIG. 2. (a) Data from a doubly clamped, $n^{+} \mathrm{Si}$ beam resonating at 25.598 $\mathrm{MHz}$ with a $Q \approx 3 \times 10^{4}$ measured in reflection (upper curves) and in bridge configurations for magnetic field strengths of $B=0,2,4,6 \mathrm{~T}$. The drive voltages are equal. The background is reduced by a factor of $\sim 200$ in the bridge measurements. The phase of the resonance in the bridge measurements can be shifted $180^{\circ}$ with respect to the drive signal (see Fig. 3). (b) The amplitude of the broadband transfer functions, $H(\omega)=V_{0}(\omega) / V_{\text {in }}(\omega)$ for both configurations. The data indicate a background reduction of at least $20 \mathrm{~dB}$ and capacitive coupling between the actuation-detection ports in the bridge circuit.

careful design of the circuit layout and the bonding pads, such problems can be minimized. Even further signal improvements can be obtained by addressing the significant impedance mismatch, $R_{e} \gg R_{L}$, between the output impedance, $R_{e}$, and the amplifier input impedance, $R_{L}$. In the measurements displayed in Fig. 2(a), this mismatch caused a signal attenuation estimated to be of order $\sim 40 \mathrm{~dB}$.

Our measurements on doped NEMS offer insight into energy dissipation mechanisms in NEMS, especially those arising from surfaces and surface adsorbates. In the frequency range investigated, $10 \mathrm{MHz}<f_{0}<85 \mathrm{MHz}$, the measured $Q$ factors of $2.2 \times 10^{4}<Q<8 \times 10^{4}$ in $n^{+}$Si beams is a factor of 2-5 higher than those obtained from metallized beams. ${ }^{15}$ Both metallization layers ${ }^{16}$ and impurity dopants ${ }^{3}$ can make an appreciable contribution to the energy dissipation. Our measurements on NEMS seem to confirm that metallization overlayers can significantly reduce $Q$ factor. The high $Q$ factors attained and the metal free surfaces make doped NEMS excellent tools for the investigation of small energy dissipation changes due to surface adsorbates and defects. In fact, efficient in situ resistive heating in doped beams through $R_{e}$ has been shown to facilitate thermal annealing ${ }^{17}$ and desorption of surface adsorbates-yielding even higher $Q$ factors.

In summary, we have developed a broadband, balanced radio frequency bridge technique for detection of small NEMS displacements. This technique may prove useful for other high frequency high impedance applications such as
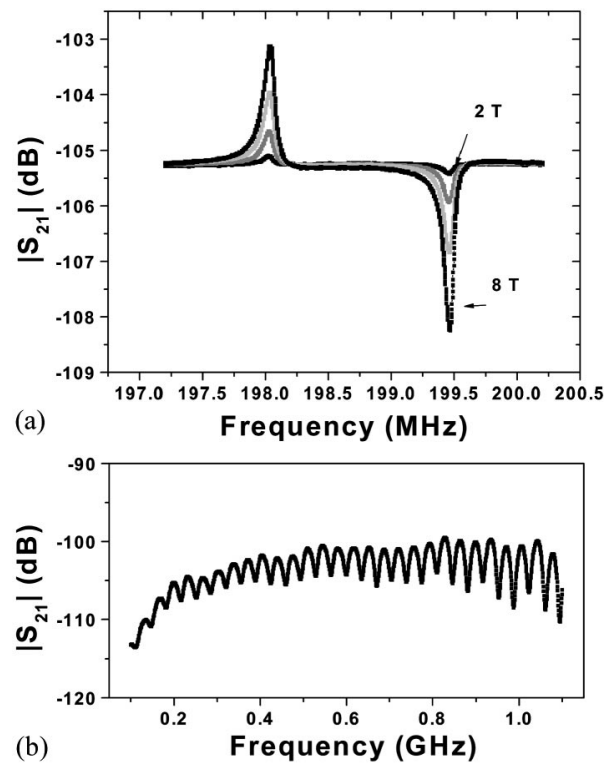

FIG. 3. Narrow band (a) and broadband (b) transfer function $\left(S_{21}\right)$ amplitudes from metallized $\mathrm{SiC}$ beams in bridge configuration. The narrowband response (a) is measured for different magnetic field strengths of $B$ $=2,4,6,8 \mathrm{~T}$ and shows two well-separated resonances at 198.00 and 199.45 MHz, respectively, with $Q \sim 10^{3}$. The broadband response (b) at $B=0 \mathrm{~T}$ shows the significant background nulling attainable in bridge measurements. We estimate that a reflection measurement on this system would produce $\left|V_{0} / V_{\text {in }}\right| \sim-20 \mathrm{~dB}$ for $\omega \approx \omega_{0}$.

piezoresistive displacement detection. The technique, with its advantages, has enabled electronic measurements of NEMS resonances otherwise essentially unmeasurable.

The authors gratefully acknowledge support from DARPA MTO/MEMS under Grant No. DABT63-98-1-0012 and technical assistance from D. Harrington.

${ }^{1}$ M. L. Roukes, Phys. World 14, 25 (2001).

${ }^{2}$ D. S. Greywall, B. Yurke, P. A. Busch, A. N. Pargellis, and R. L. Willett, Phys. Rev. Lett. 72, 2992 (1992).

${ }^{3}$ R. E. Mihailovich and J. M. Parpia, Phys. Rev. Lett. 68, 3052 (1994).

${ }^{4}$ W. C. Tang, T. C. H. Nguyen, M. W. Judy, and R. T. Howe, Sens. Actuators A 21, 328 (1990).

${ }^{5}$ M. Tortonose, R. C. Barret, and C. F. Quate, Appl. Phys. Lett. 62, 834 (1993).

${ }^{6}$ A. N. Cleland and M. L. Roukes, Appl. Phys. Lett. 69, 2653 (1996).

${ }^{7}$ A. N. Cleland and M. L. Roukes, Sens. Actuators A 72, 256 (1999).

${ }^{8}$ To simplify the length of the transmission line, $l$, between the NEMS and the measurement point has been set to $l \approx \lambda / 2$ where $\lambda$ is the drive wavelength. Also, the reflection coefficient, $\Gamma$, from the NEMS, defined as the ratio of the amplitudes of reflected to incident voltages, is taken as unity. Experimentally, $l$ is readily adjustable and $\Gamma \sim 1$ with $R_{e} \sim 100 \Omega$ $-1 \mathrm{k} \Omega$.

${ }^{9}$ Y. T. Yang, K. L. Ekinci, X. M. H. Huang, L. M. Schiavone, M. L. Roukes, C. A. Zorman, and M. Mehregany, Appl. Phys. Lett. 78, 162 (2001).

${ }^{10}$ When $\Gamma \neq 1, V_{0} \simeq \Gamma V_{\text {in }}\left(R_{e}+Z_{m}(\omega)\right) /\left(R_{L}+R_{e}\right)$, giving a correction to the background on the order of $-20 \log \Gamma \mathrm{dB}$.

${ }^{11}$ Y. T. Yang, K. L. Ekinci, and M. L. Roukes (unpublished).

${ }^{12}$ L. Pescini, A. Tilke, R. H. Blick, H. Lorenz, J. P. Kotthaus, W. Eberhardt, and D. Kern, Nanotechnology 10, 418 (1999).

${ }^{13}$ S. M. Sze, Physics of Semiconductor Devices (Wiley, New York, 1981).

${ }^{14}$ Replacing $R_{e}$ with $R_{e}+R_{S}$ would produce the more general form.

${ }^{15}$ We have qualitatively compared $Q$ factors of eight metallized and 14 doped $\mathrm{Si}$ beams measured in different experimental runs, spanning the indicated frequency range.

${ }^{16}$ X. Liu, E. Thompson, B. E. White, Jr., and R. O. Pohl, Phys. Rev. B 59, 11767 (1999).

${ }^{17}$ K. Y. Yasumura, T. D. Stowe, E. M. Chow, T. Pfafman, T. W. Kenny, B. C. Stipe, and D. Rugar, J. Microelectromech. Syst. 9, 117 (2000). 